\title{
Electronic structure of a subnanometer wide bottom-up fabricated graphene nanoribbon: End states, band gap, and dispersion
}

\author{
C. Bronner, ${ }^{1}$ F. Leyssner, ${ }^{1}$ S. Stremlau, ${ }^{1}$ M. Utecht,${ }^{2}$ P. Saalfrank,${ }^{2}$ T. Klamroth, ${ }^{2}$ and P. Tegeder ${ }^{1, *}$ \\ ${ }^{1}$ Fachbereich Physik, Freie Universität Berlin, Arnimallee 14, 14195 Berlin, Germany \\ ${ }^{2}$ Universität Potsdam, Institut für Chemie, Theoretische Chemie, Karl-Liebknecht-Straße 24-25, 14476 Potsdam, Germany
}

(Received 10 November 2011; published 23 August 2012)

\begin{abstract}
Angle-resolved two-photon photoemission and high-resolution electron energy loss spectroscopy are employed to derive the electronic structure of a subnanometer atomically precise quasi-one-dimensional graphene nanoribbon (GNR) on $\mathrm{Au}(111)$. We resolved occupied and unoccupied electronic bands including their dispersion and determined the band gap, which possesses an unexpectedly large value of $5.1 \mathrm{eV}$. Supported by density functional theory calculations for the idealized infinite polymer and finite size oligomers, an unoccupied nondispersive electronic state with an energetic position in the middle of the band gap of the GNR could be identified. This state resides at both ends of the ribbon (end state) and is only found in the finite sized systems, i.e., the oligomers.
\end{abstract}

DOI: 10.1103/PhysRevB.86.085444

PACS number(s): 73.20.Hb, 61.48.Gh, 71.15.Mb, 81.07.Gf

\section{INTRODUCTION}

Along with the great interest in graphene that has emerged in recent years, ${ }^{1}$ other low-dimensional carbon-based systems have attracted attention. One such system are graphene nanoribbons (GNRs). These flat, narrow structures are quasione-dimensional and have been the subject of numerous theoretical ${ }^{2-5}$ as well as some experimental studies ${ }^{6-9}$ because (unlike graphene itself) they exibit a band gap tunable over a wide range. This is important for their possible implementation in nanoscale devices such as transistors or transparent electrodes, whereas they maintain the large carrier mobility found in graphene sheets. ${ }^{1,7}$ In addition to the width, one of the parameters influencing the band gap (and the band dispersion along the ribbon axis) is the edge shape. Depending on the corresponding crystallographic direction in graphene, the ribbon edge's character varies from armchair to zigzag quasicontinuously, ${ }^{10}$ much like in carbon nanotubes. The band gap of GNRs is inversely proportional to the ribbon width, ${ }^{3,10}$ yielding gap sizes up to several electron volts in the subnanometer regime. Although for a wide range of ribbon widths this inverse relationship has been experimentally observed $^{6,7}$ and complies with theory, atomically small widths $(<1 \mathrm{~nm})$ and the corresponding gaps of more than $1 \mathrm{eV}$ have not been observed, which is due to the preparation methods employed. Another property of interest is the carrier mobility in GNRs, which manifests itself, e.g., in the one-dimensional band structure of the ribbons which in first approximation resembles the band structure of graphene projected onto the respective crystallographic direction. ${ }^{2}$ However, the lateral confinement of the electrons in the ribbon alters the dispersion and, in particular, opens a gap at the Dirac point. Theory has dealt in great detail with the dispersion in GNRs, but there have been no corresponding experiments published so far.

Synthesizing suitable GNRs is challenging, particularly for established methods such as lithography ${ }^{6}$ and unzipping of carbon nanotubes ${ }^{11}$ because in both cases the widths are usually larger than $10 \mathrm{~nm}$ and because of defects at the edges influencing the electronic structure. A powerful alternative in the fabrication of nanostructures is on-surface synthesis, ${ }^{12}$ which has been successfully applied for the generation of atomically precise GNRs. ${ }^{8}$ Thereby, the GNR is formed via a surface-assisted and thermally activated ${ }^{13}$ two-step process [see Fig. 1(a)], in which the adsorbed precursor molecule $10,10^{\prime}$-dibromo-9,9'-bianthryl is dehalogenated followed by $\mathrm{C}-\mathrm{C}$ coupling to form a polymer, and finally cyclodehydrogenation yields the defect-free armchair GNRs with a well-defined width of $0.7 \mathrm{~nm}$.

In this paper, we utilize angle-resolved two-photon photoemission (2PPE) and high-resolution electron energy loss spectroscopy (HREELS) to determine the electronic band structure, i.e., unoccupied and occupied electronic states as well as the band gap and dispersion for the GNR shown in Fig. 1(a) adsorbed on $\mathrm{Au}(111)$. Supported by density functional theory (DFT) calculations, we demonstrate that the band gap of the ribbon is surprisingly large, namely $5.1 \mathrm{eV}$, and contradicts all calculated values known from the literature. ${ }^{2-5}$ An unoccupied electronic state is found in the band gap which possesses no dispersion and originates from the molecular frontier orbitals localized at the ends of the GNR which have a finite length.

\section{RESULTS AND DISCUSSION}

The thermally activated surface-supported formation steps of the GNR on $\mathrm{Au}(111)$ can be followed nicely by angleresolved vibrational HREELS, since well-defined and pronounced changes in the vibrational spectrum are observed for each step. Figure 1(b) shows the data for both the polymeric phase and the aromatic nanoribbons. In the specular spectrum of the linear polymer, we observe several out-ofplane molecular vibrations, namely the $\mathrm{C}-\mathrm{H}$ wagging mode $[\gamma(\mathrm{C}-\mathrm{H})]$ at $758 \mathrm{~cm}^{-1}$, an edge mode at $594 \mathrm{~cm}^{-1}$, and the phenyl ring torsion modes $[\tau(\mathrm{C}-\mathrm{C})]$ at $424 \mathrm{~cm}^{-1}$. All these modes show a drastic decrease in the spectrum measured in off-specular geometry, indicating that their intensities are predominantly originating from dipole scattering, i.e., they are dipole-active. Thus, the corresponding dipole moment changes during vibration can be inferred to lie perpendicular to the surface. This points toward a mainly flat adsorption geometry of the phenyl rings in the polymeric phase. Due 

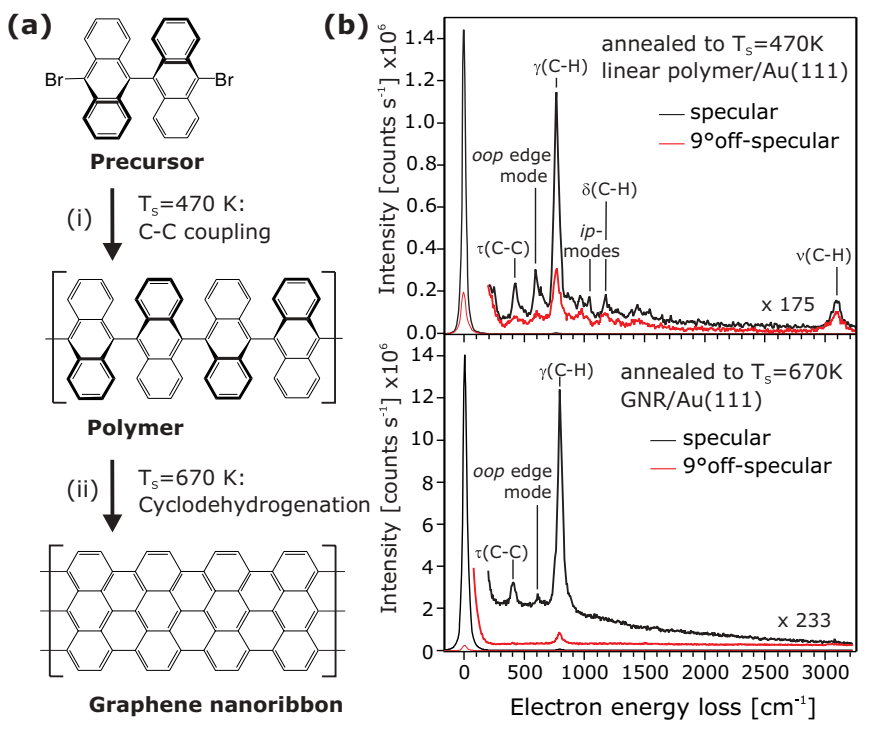

FIG. 1. (Color online) (a) Scheme of the surface-supported fabrication of graphene nanoribbons (GNRs) via a two-step process: (i) dehalogenation of precursor molecule 10,10'-dibromo-9, $9^{\prime}$ bianthryl followed by a C-C coupling and (ii) cyclodehydrogenation. (b) Changes in the vibrational HREEL spectrum observed during the cyclodehydrogenation step from the linear polymer to the nanoribbon measured with a primary electron energy of $3.5 \mathrm{eV}$.

to the single $\mathrm{C}-\mathrm{C}$ bonds connecting the building blocks of the polymer and the steric hinderance of the $\mathrm{C}-\mathrm{H}$ groups, they are slightly tilted as observed in scanning tunneling microscopy (STM). ${ }^{8}$ Indeed, at $3055 \mathrm{~cm}^{-1}$ the $\mathrm{C}-\mathrm{H}$ stretch mode $[v(\mathrm{C}-\mathrm{H})]$ shows a weak dipole activity. Accordingly, this vibration exhibits a component of the dipole moment change parallel to the surface normal which is due to a tilting. Hence, our data fully support the adsorption geometry reported by Cai et al. ${ }^{8}$ Upon cyclodehydrogenation and thus formation of the nanoribbons, the vibrational spectrum is changed significantly. The number of molecular vibrations found in the spectrum is reduced to three modes. The out-of-plane modes, the phenyl ring torsion mode $\tau(\mathrm{C}-\mathrm{C})\left(404 \mathrm{~cm}^{-1}\right)$, the edge mode $\left(609 \mathrm{~cm}^{-1}\right)$, and the bending mode $\gamma(\mathrm{C}-\mathrm{H})$ at $793 \mathrm{~cm}^{-1}$ are all purely dipole-active. This clearly demonstrates that all phenyl rings are now orientated parallel (flat-lying) to the surface as expected for the aromatic GNR. By adsorption of xenon onto the so prepared GNR-covered surface, we may roughly infer the coverage of the nanoribbons on the gold surface: in temperature-programmed desorption, two desorption peaks are observed which do not grow in intensity as the layer thickness is increased to very high coverages. These features are assigned to Xe desorbing from both between and on top of the nanoribbons, respectively. From their peak intensities, we can roughly infer a coverage of approximately $2 / 3 \mathrm{ML}$.

Electronic HREELS as well as 2PPE are employed to gain insight into the occupied and unoccupied GNR-derived electronic states and therefore the band gap. Figure 2(a) shows the result obtained from electronic HREELS using a primary electron energy of $E_{0}=15 \mathrm{eV}$. A double-peak structure is observed which we fitted with two Gaussian peaks yielding transition energies of $2.6 \mathrm{eV}$ and $3.1 \mathrm{eV}$, respectively. An exemplary two-color 2PPE spectrum recorded with $2.26 \mathrm{eV}$
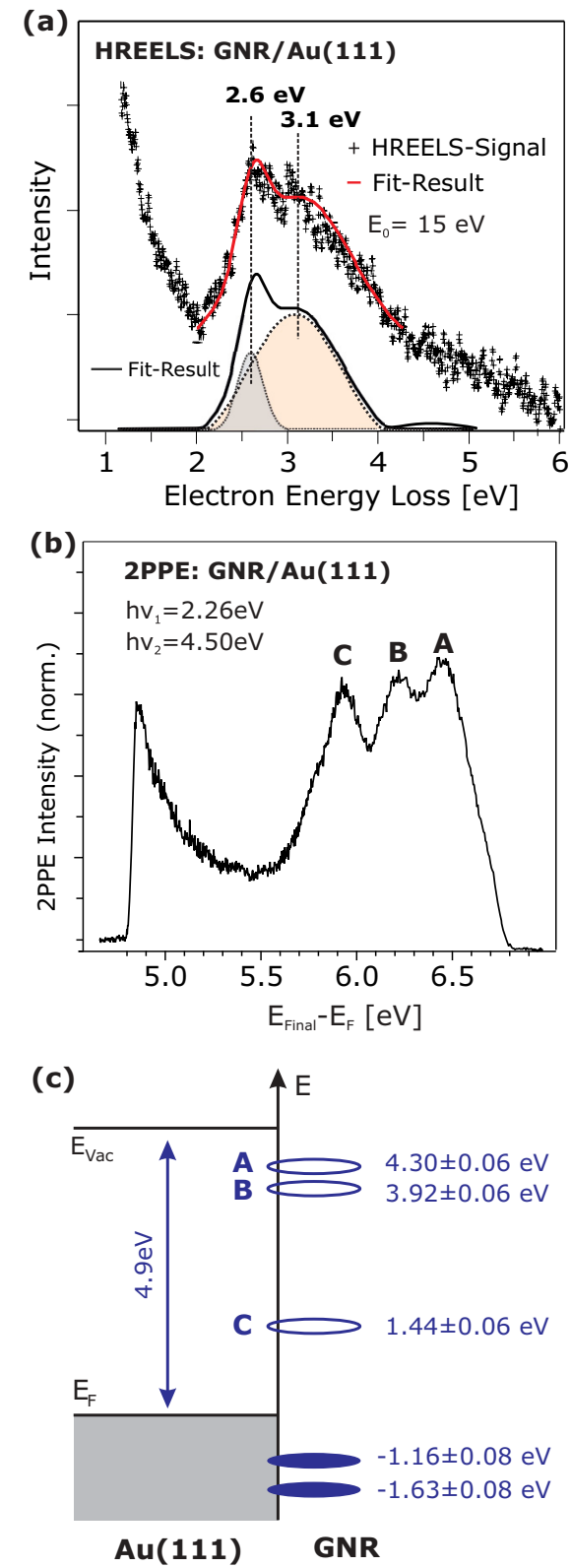

FIG. 2. (Color online) (a) Electronic HREEL spectrum of the GNR-covered gold surface recorded at a primary electron energy of $15 \mathrm{eV}$. Two electronic transitions are observed which are fitted using two Gaussian peaks. (b) Two-color 2PPE spectra recorded at photon energies of $2.26 \mathrm{eV}$ and $4.50 \mathrm{eV}$ of GNR/Au(111). (c) Energetic position of GNR-derived electronic states. The Fermi level of $\mathrm{Au}(111)$ serves as reference.

and $4.50 \mathrm{eV}$ photons is displayed in Fig. 2(b). Several peaks are observed, and on the basis of photon-energy-dependent measurements ${ }^{14,15}$ they can be related to photoemission from unoccupied intermediate states. The peaks labeled with $\mathrm{A}$ and $\mathrm{B}$ are both populated with the $4.50 \mathrm{eV}$ photons and probed by $h v=2.26 \mathrm{eV}$, thus they are located at $4.30 \pm 0.06 \mathrm{eV}$ and $3.92 \pm 0.06 \mathrm{eV}$, respectively, with respect to the Fermi level $\left(E_{F}\right)$. Note that the energetic positions given here are average values based on several measurements. The peak labeled $\mathrm{C}$ originates from a state which is probed by the $4.50 \mathrm{eV}$ photons, therefore it possesses an energetic position of 
$1.44 \pm 0.06 \mathrm{eV}$ with respect to $E_{F}$. Combining the HREELS transition energies with the 2PPE results, we conclude that there are two occupied electronic states lying at $-1.16 \pm$ $0.08 \mathrm{eV}$ and $-1.63 \pm 0.08 \mathrm{eV}$ with respect to $E_{F}$. Since these energies comply neither with the bulk band structure of $\mathrm{Au}(111)$ nor with the Shockley surface state, these occupied states can be assigned to the GNR. Figure 2(c) summarizes the binding energies of all electronic states observed in the present study. On the basis of the obtained data, one would establish a band gap of $2.6 \mathrm{eV}$ as found as the lowest transition in the electronic HREELS measurement, which is in reasonable agreement with a recent measurement of the band gap on spatially aligned GNR using ultraviolet and inverse photoemission. ${ }^{16}$ However, for an unambiguous identification of the band gap, one needs further information on the nature of the unoccupied bands (states). To gain deeper insight into the properties, we performed dispersion measurements using angle-resolved 2PPE, which provide information about the extent of electron delocalization/localization.

As observed in STM, ${ }^{8}$ the GNRs investigated in this study are oriented randomly on the $\mathrm{Au}(111)$ surface. In our experiment, the momentum information is obtained by rotating the sample in front of the time-of-flight (TOF) spectrometer, as seen in Fig. 3(a). While all nanoribbons contribute to the signal detected in normal emission, this is not the case
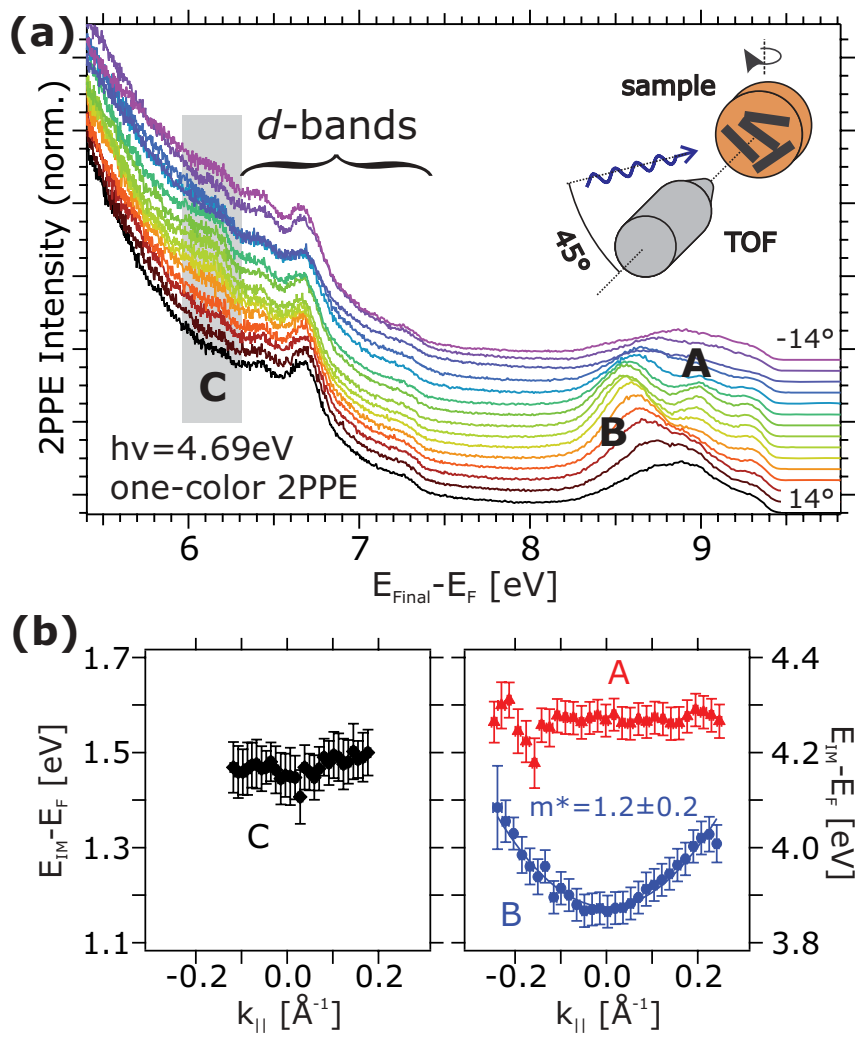

FIG. 3. (Color online) (a) Angle-resolved one-color 2PPE spectra. (Inset: Measuring geometry in angle-resolved 2PPE experiments. Among the randomly oriented GNRs on the surface, mainly those lying in the plane perpendicular to the rotation axis of the sample are detected for off-normal emission.) (b) Dispersion of the spectral feature labeled as A, B, and C. While the states A and C are localized, state B shows a strong dispersion, viz., it is delocalized. as we rotate the sample. A photoelectron emitted from the one-dimensional band structure of the GNR band has two contributions to its momentum $k_{\|}$parallel to the surface, i.e., the component along the ribbon axis (which is the quantity of interest) and a component perpendicular to it which is random (as the electrons are localized in this direction). Due to the measuring geometry, our TOF only detects photoelectrons emitted in the plane perpendicular to the rotation axis. This can either be electrons emitted along the axis of ribbons lying in this plane or electrons which (due to the random momentum perpendicular to the ribbon axis) are emitted at an angle with respect to the ribbon, which is equal to the angle between this GNR and the detection plane. In the latter case, the detected momentum will be higher than the momentum the electron had in the band, which causes the measured dispersion to be smeared out to higher $k_{\|}$, but not in the energy domain. Since for finite angles only ribbons lying perpendicular to the rotation axis can be measured, we expect a significant drop in photoemission intensity away from the $\Gamma$ point.

From the three unoccupied states observed in 2PPE, only the one labeled B shows a dispersion, viz., the states A and $\mathrm{C}$ are localized. Figure 3(a) displays a series of one-color 2PPE spectra recorded at a photon energy of $4.69 \mathrm{eV}$ for various angles between the surface normal and the TOF axis. In addition to the contributions of the gold $d$-bands, the peaks labeled A, B, and C are seen. While B exhibits a strong dispersion around the $\Gamma$ point, states $\mathrm{A}$ and $\mathrm{C}$ show no dispersion [see Fig. 3(b)]. The parabolic behavior of state B complies qualitatively with all theoretical calculations on armchair nanoribbons. The effective mass $\left(m^{*}\right)$ of $1.2 m_{e}$ is significantly higher than that generally predicted by theoretical models for a variety of GNRs. ${ }^{17,18}$

However, most surprisingly, the lowest unoccupied state located at $1.44 \mathrm{eV}$ above $E_{F}$ (peak C) exhibits no dispersion, therefore we assign the first dispersive state (state B) above the Fermi level, which possesses an energetic position of $3.92 \mathrm{eV}$, to the conduction band of the GNR. Thus, with the valence band located at $-1.16 \mathrm{eV}$ with respect to $E_{F}$, the band gap is $5.1 \mathrm{eV}$. This value is significantly higher than the calculated values lying in the range between 1.5 and $2.1 \mathrm{eV}$, depending on the applied method. ${ }^{8}$ For use in technological applications, the size of the band gap plays a key role; the one we observe for the studied armchair GNR is too large, for instance, to use in a nanowire or even a transistor, where a small gap is preferred.

To understand the nature of the localized state $C$, we performed DFT calculations for the idealized infinite polymer and finite size oligomers employing the Perdew-BurkeErnzerhof generalized gradient approximation (PBE GGA) functional and the range separated hybrid functional Coulombattenuating method - Becke, Lee-Yang-Parr (CAM-B3LYP), which is particularly suitable for long chain molecular systems (polyene), ${ }^{19}$ together with the $6-311 \mathrm{G}^{* *}$ basis set as implemented in the GAUSSIAN09 program package. ${ }^{20}$ All calculations were done for the GNR alone, i.e., without the supporting $\mathrm{Au}(111)$ surface, and the geometry of the GNR was fully optimized. For the periodic calculations, a $k$-point grid with 240 points in the first Brillouin zone (1BZ) and a real space cutoff of 600 bohrs were used. The PBE results obtained here compare very well with calculations reported by Cai 

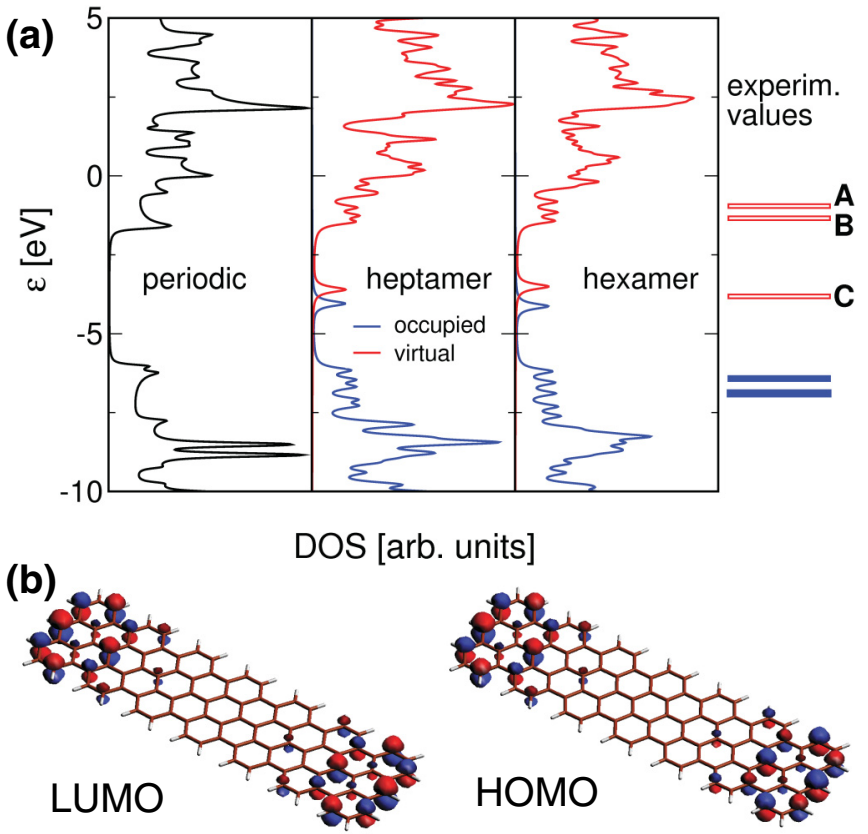

FIG. 4. (Color online) (a) Density of states obtained by Lorentzian broadening for the periodic case (black line) and for the occupied (blue lines) and virtual orbitals (red lines) of the heptamer and the hexamer together with the experimental energies, which have been aligned to the middle of the band gap of the periodic GNR. (b) HOMO and LUMO for the heptamer, which are localized at the ends of the oligomer (all calculations at the CAM-B3LYP/6-311G** level of theory).

and co-workers. ${ }^{8}$ Furthermore, for the periodic calculations, the geometry of the GNR depends only very weakly on the choice of the functional. For instance, we obtain a lattice constant of $4.310 \AA$ for PBE, and for CAM-B3LYP we get $4.277 \AA$. However, the band gap changes drastically due to the admixture of exact exchange in the CAM-B3LYP functional. Using the PBE functional results in a gap of $1.54 \mathrm{eV}$, while for CAM-B3LYP a gap of $4.35 \mathrm{eV}$ is obtained. The same qualitative trend is observed when using $\mathrm{PBE} 0,{ }^{21}$ i.e., $\mathrm{PBE}$ with an admixture of exact exchange, where we obtain a band gap of $2.7 \mathrm{eV}$. Also, it has been shown that CAM-B3LYP is especially suitable for structural and electronic properties of large unsaturated hydrocarbons, ${ }^{22}$ which is in line with the much better agreement of CAM-B3LYP with the experimental results reported here.

However, apart from the wider band gap and the larger dispersion, the band structures for the PBE and CAM-B3LYP are qualitatively similar and no indication of a nondispersive state corresponding to the localized state $\mathrm{C}$ could be found in the periodic calculations for the idealized infinite polymer. Therefore, we carried out additional calculations for oligomers up to the heptamer, where the oligomers were saturated with hydrogens at both ends and again a full geometry optimization was performed. To compare the electronic structure of the oligomers with that of the periodic structure, we computed the density of states (DOS) by a simple Lorentzian-broadening procedure, where every Kohn-Sham orbital energy or every crystal orbital energy within the $1 \mathrm{BZ}$ is represented by a Lorentzian with a width $\Gamma=0.2 \mathrm{eV}$ for the oligomers and $\Gamma=0.1 \mathrm{eV}$ for the periodic case. Figure 4(a) shows the DOS for the periodic case (black line) and for the occupied (blue lines) and virtual, i.e., unoccupied, orbitals (red lines) of the heptamer and the hexamer using the CAM-B3LYP functional. As one can see, the DOS for the valence and the conduction bands are quite similar. The same holds for the geometric structure, as we get a length of $4.278 \AA$ for the monomer unit in the middle of the heptamer, which is nearly identical to the lattice constant of $4.277 \AA$ in the periodic case.

However, for the electronic structure of the oligomers, we observe two states inside the "band gap," namely the highest occupied molecular orbital (HOMO) and the lowest unoccupied molecular orbital (LUMO). These states are localized at both ends of the oligomers, as shown in Fig. 4(b) for the heptamer. Furthermore, the energy difference between these two localized states decreases for longer chain lengths. In the experiments, the GNRs have a length that corresponds to dozens of units (monomer length $0.43 \mathrm{~nm}$ ), ${ }^{8}$ for which a nonresolvable energy difference between the two localized states is to be expected. In addition, the HOMO of the free GNR should also be unoccupied when adsorbed at the $\mathrm{Au}(111)$ surface, as its energy is above the Fermi level. Therefore, these localized states can be identified as state $\mathrm{C}$ comparing the calculated energies with experimentally determined energies, which are indicated on the right side of Fig. 4(a).

\section{CONCLUSION}

In conclusion, using two complementary surface-sensitive experimental methods, namely HREELS and 2PPE, in combination with DFT calculations, we were able to probe key electronic properties of a defect-free graphene nanoribbon. We determined the band gap to be $5.1 \mathrm{eV}$, which is surprisingly high since previous calculations predicted much lower values. A nondispersive unoccupied electronic state of the GNR located in the band gap originates from both the HOMO and LUMO of the ribbons with a finite length (oligomer). These states are localized at both ends of the ribbon, thus we named them "end states". A controlled modification of the precursor molecule with suitable substituents or by doping may certainly pave the way to create precise nanoribbons with lower band gaps, appropriate for applications.

\section{ACKNOWLEDGMENTS}

We gratefully acknowledge financial support by the FU Berlin through the Focus Area "NanoScale" and by the DFG through the collaborative research center SFB 658. We thank Cornelius Gahl (FU Berlin) for very fruitful discussions.

\footnotetext{
*petra.tegeder@physik.fu-berlin.de

${ }^{1}$ A. K. Geim and K. S. Novoselov, Nat. Mater. 6, 183 (2007).

${ }^{2}$ K. Nakada and M. Fujita, Phys. Rev. B 54, 17954 (1996).
}

${ }^{3}$ V. Barone, O. Hod, and G. E. Scuseria, Nano Lett. 6, 2748 (2006).

${ }^{4}$ Y.-W. Son, M. L. Cohen, and S. G. Louie, Phys. Rev. Lett. 97, 216803 (2006). 
${ }^{5}$ A. J. Chaves, G. D. Lima, W. de Paula, C. E. Cordeiro, A. Delfino, T. Frederico, and O. Oliveira, Phys. Rev. B 83, 153405 (2011).

${ }^{6}$ M. Y. Han, B. Özyilmaz, Y. Zhang, and P. Kim, Phys. Rev. Lett. 98, 206805 (2007).

${ }^{7}$ X. Li, X. Wang, L. Zhang, S. Lee, and H. Dai, Science 319, 1229 (2008).

${ }^{8}$ J. Cai et al., Nature (London) 466, 470 (2010).

${ }^{9}$ C. Tao et al., Nat. Phys. 7, 616 (2011).

${ }^{10}$ M. Ezawa, Phys. Rev. B 73, 045432 (2006).

${ }^{11}$ D. V. Kosynkin, A. L. Higginbotham, A. Sinitskii, J. R. Lomeda, A. Dimiev, B. K. Price, and J. M. Tour, Nature (London) 458, 872 (2009).

${ }^{12}$ L. Grill, M. Dyer, L. Lafferentz, M. Persson, M. V. Peters, and S. Hecht, Nat. Nanotech. 2, 687 (2007).

${ }^{13}$ J. Björk, S. Stafström, and F. Hanke, J. Am. Chem. Soc. 133, 14884 (2011).
${ }^{14}$ S. Hagen, Y. Lou, R. Haag, M. Wolf, and P. Tegeder, New J. Phys. 12, 125022 (2010).

${ }^{15}$ C. Bronner, M. Schulze, S. Hagen, and P. Tegeder, New J. Phys. 14, 043032 (2012).

${ }^{16}$ S. Linden, D. Zhong, A. Timmer, N. Aghdassi, J. H. Franke, H. Zhang, X. Feng, K. Müllen, H. Fuchs, L. Chi, and H. Zachariasm, Phys. Rev. Lett. 108, 216801 (2012).

${ }^{17}$ G. Wang, Phys. Chem. Chem. Phys. 13, 11939 (2011).

${ }^{18}$ L. Yang, C.-H. Park, Y.-W. Son, M. L. Cohen, and S. G. Louie, Phys. Rev. Lett. 99, 186801 (2007).

${ }^{19}$ T. Yanai, D. Tew, and N. Handy, Chem. Phys. Lett. 393, 51 (2004).

${ }^{20}$ GaUssian09, Revision A.02, M. J. Frisch et al. (Gaussian, Inc., Wallingford, CT, 2009).

${ }^{21}$ C. Adamo and V. Barone, J. Chem. Phys. 110, 6158 (1999).

${ }^{22}$ M. J. G. Peach et al., J. Phys. Chem. A 111, 11930 (2007). 